\title{
Évaluation préliminaire de la motivation d'étudiants en cours d'externat à remplir les formulaires d'évaluation en ligne des stages hospitaliers
}

\author{
Preliminary evaluation of clerk commitment in participating in online \\ clerkship evaluation forms
}

\author{
Audrey FARRUGIA ${ }^{1}$, Thierry PELACCIA ${ }^{2,3}$, Thierry POTTECHER ${ }^{3,4}$ et Bertrand LUDES ${ }^{1}$ \\ 1 Institut de médecine légale, Hôpitaux Universitaires de Strasbourg, Université de Strasbourg, Strasbourg, France \\ 2 Service d'aide médicale urgente (SAMU 67) et Centre d'enseignement des soins d'urgence (CESU 67), \\ Hôpitaux universitaires de Strasbourg, Strasbourg, France \\ 3 Laboratoire de recherche en pédagogie des sciences de la santé, Faculté de médecine de Strasbourg, Strasbourg, France \\ 4 Pôle d'anesthésie-réanimation chirurgicale, Hôpitaux Universitaires de Strasbourg, Strasbourg, France
}

Manuscrit soumis le 29 mars 2011 ; commentaires éditoriaux formulés aux auteurs le 13 juillet 2011 et le 18 juillet 2012 ; accepté pour publication le 20 juillet 2012

\section{Mots-clés}

Motivation ; évaluation ; autodétermination; stages hospitaliers.

Résumé - Contexte : L'implication active des étudiants est un élément-clé de la démarche d'évaluation de la qualité des stages hospitaliers. L'exhaustivité des réponses au formulaire d'évaluation des stages hospitaliers par les étudiants du deuxième cycle de la faculté de médecine de l'Université de Strasbourg limite la possibilité d'exploiter ces données dans une perspective d'amélioration des stages, et amène à se questionner sur la motivation des étudiants à participer à cette évaluation. Buts : Afin d'augmenter la fiabilité de l'évaluation des stages hospitaliers, cette étude cherche à identifier les caractéristiques individuelles et le profil motivationnel des étudiants remplissant le formulaire d'évaluation des stages cliniques, au regard de la théorie de l'autodétermination et du modèle de dynamique motivationnelle développée par Viau. Méthode : 223 étudiants en médecine inscrits en troisième année du deuxième cycle des études médicales (DCEM3) ont été soumis à un questionnaire anonyme auto-administré. Résultats : Les caractéristiques individuelles des non-répondants diffèrent selon qu'il s'agisse des non-répondants au dernier formulaire ou des non-répondants à tous les formulaires pour l'année 2008-2009. Par ailleurs, la probabilité de remplir le dernier formulaire en ligne est cinq fois moindre pour un étudiant ayant une activité rémunérée et la probabilité de répondre à l'ensemble des formulaires double pour un étudiant assistant aux conférences d'internat. De plus, les motifs d'engagement des étudiants dans l'évaluation des stages cliniques sont faiblement autodéterminés. Enfin, l'analyse de la dynamique motivationnelle a permis de souligner la faible perception de la tâche et la faible perception de contrôle ressentie par les étudiants. Conclusion : Ces résultats permettent de formuler des propositions concrètes d'actions afin d'augmenter la motivation des étudiants en médecine à s'engager dans un processus d'évaluation des stages.
\end{abstract}




$\begin{array}{ll}\begin{array}{l}\text { Keywords } \\ \text { Motivation; } \\ \text { evaluation; self- }\end{array} & \text { Abstract - Context: Key to the evaluation approach of the quality of clerkship training in } \\ \text { determination; } & \text { hospitals is active student involvement. The low response rate in clinical clerkship evaluation } \\ \text { hospital clerkship } & \text { forms by medical students at the university of Strasbourg school of medicine restricted the } \\ \text { training } & \text { use of the data in view of improving training. It questions student motivation in participating } \\ \text { in evaluations. Objectives: In order to improve the reliability of clinical clerkship evalua- } & \text { tions, the study focused on identifying the individual characteristics and expectations of stu- } \\ \text { dents filling the online form from the viewpoint of the self-determination theory and the } \\ \text { model of motivational dynamics developed by Viau. Method: A survey was conducted with } \\ \text { 223 DCEM3 medical students using an anonymous self-administered questionnaire. Results: } \\ \text { The individual characteristics of non-respondents varied depending on the fact that responses } \\ \text { came from the latest online form or 2008-2009 questionnaires. In addition, the likelihood of } \\ \text { filling the latest online form was five times lower among students holding down a job while } \\ \text { the probability of responding to the 2008-2009 form doubled among students participating in } \\ \text { internship conferences. Moreover, student commitment in evaluating clinical clerkships was } \\ \text { poorly self-determined. Lastly, the study highlighted the low perceived task value and the low } \\ \text { control perception among students. Conclusion: The results offer practical suggestions desi- } \\ \text { gned to increase medical student motivation in committing to clerkship training evaluation. }\end{array}$

\section{Introduction}

L'évaluation des enseignements cliniques s'inscrit dans le cadre d'une démarche qualité mise en place dans de nombreuses universités françaises. Elle répond au cadre réglementaire défini par l'article 23 de l'arrêté du 9 avril 1997 (publié au Journal Officiel du 15 avril 1997) relatif au diplôme d'études universitaires générales, à la licence et à la maitrise. À la faculté de médecine de l'Université de Strasbourg, la mise en place et la coordination de cette démarche d'évaluation est assurée depuis le mois d'octobre 2003 par la cellule d'évaluation des enseignements théoriques et cliniques (CEETC), rattachée au département de pédagogie de la faculté. Cette dernière a créé un formulaire d'évaluation des stages hospitaliers devant être rempli en ligne par les étudiants de deuxième cycle au terme des stages d'une durée supérieure à un mois. Les résultats sont transmis à la CEETC et aux référents pédagogiques de chaque pôle hospitalier pour qu'ils en assurent l'analyse et la diffusion auprès des médecins qui prennent en charge les étudiants. Le défaut d'exhaustivité des réponses à ce formulaire - $60 \%$ de retour en 2008 - limite l'exploitation de ces données et amène à se questionner sur la motivation des étudiants à participer à l'évaluation des stages.
Ces constatations nous ont conduits à examiner l'hypothèse que le manque d'engagement de certains étudiants dans la tâche d'évaluation des stages hospitaliers pourrait être lié à un défaut de motivation. De plus, il s'avère également intéressant de rechercher si des éléments contextuels (tels que des contraintes matérielles) pouvaient constituer des freins à répondre au formulaire d'évaluation.

Par conséquent, l'objectif principal de notre travail est de définir les caractéristiques individuelles et le profil motivationnel des étudiants remplissant - ou non - le formulaire d'évaluation des stages et d'identifier les facteurs contextuels ou motivationnels susceptibles de constituer des leviers d'action pour la faculté de médecine, dans une perspective d'incitation des étudiants à s'inscrire dans la démarche d'évaluation des stages hospitaliers.

\section{Cadre conceptuel}

\section{Définition du concept de motivation}

La motivation est un concept polymorphe qui a fait l'objet de nombreuses approches théoriques depuis le début du XX $\mathrm{XX}^{\mathrm{e}}$ siècle. Selon Vallerand et Thill ${ }^{[1]}$, la 
motivation est définie comme « le construit hypothétique utilisé afin de décrire les forces internes et/ou externes produisant le déclenchement, la direction, l'intensité et la persistance du comportement ». La motivation constitue ainsi le moteur de nos comportements, dont l'origine peut être intrinsèque et/ou extrinsèque. Dans cette étude le profil motivatonnel des étudiants a été établi en se basant sur la théorie de l'autodétermination et sur le modèle de la dynamique motivationnele de Viau.

\section{Théorie de l'autodétermination}

La théorie de l'autodétermination-développée par les psychologues américains Deci et Ryan ${ }^{[2]}$ dans les années quatre-vingt - permet d'établir le profil motivationnel des individus en fonction de leur niveau d'autodétermination. Un niveau élevé d'autodétermination traduit un engagement libre du sujet dans une activité qu'il privilégie en raison de l'intérêt, de la satisfaction, voire du plaisir qu'elle procure. Deci et Ryan ${ }^{[2]}$ distinguent plusieurs types de comportements selon le niveau décroissant d'autodétermination ${ }^{[3]}$ : 1) la motivation intrinsèque désigne les individus pratiquant une activité pour l'intérêt, la satisfaction ou le plaisir qu'elle procure ; 2) la motivation extrinsèque par régulation identifiée concerne les sujets qui s'engagent dans une action parce qu'elle leur permettra de réaliser leurs projets et d' atteindre des objectifs professionnels ou personnels même si leur intérêt pour la tâche est faible ; 3) la motivation extrinsèque par régulation introjectée caractérise les comportements dictés par les attentes de l'environnement social des sujets et 4) la motivation extrinsèque par régulation externe correspond à des comportements influencés par la recherche des récompenses ou par l'évitement de sanctions.

La motivation intrinsèque et les différents niveaux de motivation extrinsèque sont explorés dans le questionnaire de l'étude.

\section{Dynamique motivationnelle}

Si la théorie de l'autodétermination permet d'identifier un profil motivationnel, les facteurs à l'origine de ce profil motivationnel et son évolution sont définis plus précisément grâce au modèle de la dynamique motivationnelle développé par Viau ${ }^{[4]}$. Dans une approche sociocognitive du concept de motivation, trois dimensions participent à la dynamique motivationnelle $^{[4]}:$ 1) la perception de la valeur de la tâche désigne le jugement que porte l'apprenant sur l'intérêt à réaliser une action au regard de ses objectifs et de ses attentes ; 2) la perception d'efficacité désigne le jugement que porte l'apprenant sur ses capacités à réussir ce qui lui a été demandé ; 3) la perception de contrôle désigne le niveau d'autonomie perçu par l'apprenant dans ses actions.

La perception d'efficacité n' a pas été incluse dans le questionnaire car ce concept semblait peu pertinent au vu de la problématique.

\section{Méthode \\ Population étudiée}

Cette étude descriptive a été menée auprès des étudiants, hors étudiants Erasmus, inscrits en troisième année du deuxième cycle des études médicales (DCEM3) à la faculté de médecine de Strasbourg pour l'année 2009-2010. Ce choix a été justifié par la possibilité de soumettre directement le questionnaire à cette promotion lors des travaux dirigés du module de médecine légale durant l'année universitaire 20092010. La présence à ces cours étant obligatoire, il s'agissait d'une démarche visant à recueillir l'effectif le plus large possible. Le questionnaire anonyme était distribué au début de chaque cours et les étudiants bénéficiaient d'une dizaine de minutes pour le remplir.

\section{Élaboration du questionnaire}

Un questionnaire a été construit spécifiquement pour l'étude par la CEETC. Il a fait l'objet de discussions au sein de l'équipe de recherche quant à la formulation des questions afin de répondre à nos hypothèses de 
recherche. In fine, un questionnaire comportant 30 items (27 questions fermées et trois questions ouvertes autorisant des réponses libres) et se divisant en deux parties (Annexe 1) a été élaboré.

La première partie - intitulée «questionnaire portant sur les caractéristiques individuelles » - précise :

- les caractéristiques individuelles de l'étudiant : âge, sexe ;

- le contexte de vie de l'étudiant : le type de logement, l'accès à internet au domicile, accès à une connexion wifi ;

- les caractéristiques professionnelles: l'exercice d'une activité rémunérée ;

- les caractéristiques de la scolarité : le statut de redoublant, la participation aux conférences d'internat ;

- les projets professionnels : type de spécialité envisagée (médecine générale, spécialité médicale, spécialité chirurgicale), mode d'exercice envisagé (libéral, salarié).

Cette partie permet d'explorer le contexte de vie du répondant dans l'objectif d'adapter potentiellement la manière dont le questionnaire pourra être soumis ultérieurement aux étudiants afin d'augmenter le taux de participation.

La seconde partie - intitulée «questionnaire motivation » - a été construite en se souciant d'arrimer le contenu recherché avec le cadre conceptuel. Ainsi cette partie évalue :

- le nombre d'individus déclarant avoir rempli le dernier formulaire d'évaluation de stage ;

- le nombre d'individus déclarant avoir rempli tous les formulaires d'évaluation de stage au cours de l'année précédente ;

- la motivation d'une telle démarche en différenciant

- la motivation extrinsèque de type identifiée avec l'item «pour donner son avis »;

- la motivation de type introjectée avec l'item « incité par vos délégués étudiants »;

- la motivation de type externe avec l'item «pour que le stage soit validé par la fac »;

- la perception de la valeur de la tâche et de contrôle avec les items «résultats non accessibles aux étudiants» ou «stages toujours aussi mal organisés ».

\section{Cohérence interne}

Une évaluation préliminaire du questionnaire a été réalisée auprès d'un échantillon-test de 24 étudiants inscrits en deuxième année du deuxième cycle des études médicales. Nous avons demandé aux étudiants de lister les trois concepts (synonymes) sous-tendus par chaque item. L'exploitation de ces données a consisté à répertorier les concepts cités et à calculer le taux de citation de chaque concept puis la moyenne des taux de citation par question. Dans le but d'augmenter la cohérence interne du questionnaire, le questionnaire initial a été modifié en tenant compte des résultats de cette évaluation. En effet, le sens de certaines questions a été précisé en rajoutant en italique le concept dont le taux de citation était le plus élevé. À titre d'exemple, pour l'item «vous l'avez fait incité(e) par vos délégués étudiants », deux concepts opposés ont été cités par les étudiants à savoir «encouragé » et «menacé». Afin que le sens compris par les étudiants soit bien celui de l'encouragement positif, il a été rajouté dans le questionnaire les termes «encouragé/motivé » à la fin de l'item.

\section{Variables d'étude}

Dans cette étude, les variables à expliquer sont « le fait d'avoir rempli le dernier formulaire en ligne » et « le fait d'avoir rempli tous les formulaires en ligne au cours de l'année 2008-2009».

Les variables explicatives étudiées sont les suivantes:

- variable personnelle quantitative : âge.

- variable personnelle qualitative : sexe.

- variable portant sur le contexte de vie de l'étudiant : accès à internet à domicile, accès à une connexion wifi, type de logement (chez les parents, seul, colocation ou cité universitaire).

- variable professionnelle : exercice d'une activité rémunérée.

- variable portant sur la scolarité : statut de redoublant, participation aux conférences d'internat.

- variables portant sur le projet professionnel: type de spécialité envisagée (médecine générale, 
spécialité médicale, spécialité chirurgicale), mode d'exercice envisagé (libéral, salarié).

\section{Analyse statistique}

Les différents items des questionnaires ont été codés pour les besoins de l'exploitation statistique. Le traitement statistique a tenu compte des critères de validité exigés par les tests. Pour la variable quantitative, à savoir l'âge, le test de Mann et Withney a été employé. Les résultats ont été donnés sous forme de moyenne et d'écart type. Pour les variables binaires, le test du $\chi^{2}$ de Pearson a été utilisé en analyse univariée afin d'analyser : a) la relation entre les caractéristiques individuelles des répondants (variables explicatives précédemment citées) et le fait d'avoir renvoyé le dernier formulaire en ligne ; b) la relation entre les caractéristiques individuelles des répondants (variables explicatives précédemment citées) et le fait d'avoir rempli tous les formulaires en ligne ; c) la relation entre les réponses au questionnaire de motivation et le fait d'avoir rempli tous les formulaires en ligne.

L'analyse multivariée par régression logistique n'a inclus que les variables explicatives pour lesquelles une différence statistiquement significative avait été retrouvée par l'analyse univariée. Le seuil de significativité est de 0,05 pour l'ensemble des tests.

L'analyse statistique a été réalisée à l' aide du logiciel STAT EL.

\section{Résultats}

L'analyse porte sur les 223 étudiants présents lors des travaux dirigés du module de médecine légale durant l'année universitaire 2009-2010 auxquels le questionnaire auto-administré a pu être soumis.

\section{Questionnaire portant sur les caractéristiques} individuelles

\section{Caractéristiques individuelles de la population étudiée}

Au sein de la population étudiée, on note une prédominance féminine avec un sexe ratio de 1,9 femme pour un homme. La moyenne d'âge est de 23,2 ans ( $\pm 1,9$ année) avec des extrêmes de 20 à 39 ans. Seuls $3 \%(n=6)$ des répondants sont des redoublants. La majorité des répondants soit $53 \%(n=118)$ vit seule dans un studio ou un appartement. La majorité des répondants soit $88,7 \%(n=198)$ possède un accès internet à domicile. Une minorité d'étudiants soit $9,8 \% \quad(n=22)$ exerce une activité rémunérée. Concernant la spécialité envisagée par les étudiants, $40 \%$ d'entre eux $(n=91)$ se destinent à une spécialité médicale, $24 \%(n=54)$ vers la médecine générale et $12 \% \quad(n=28)$ vers une spécialité chirurgicale. Concernant le mode d'exercice, la majorité des répondants soit $45 \%(n=102)$ souhaite avoir un exercice de type libéral contre $16,5 \%(n=37)$ un exercice salarié. Un peu plus d'un tiers $(37 \%, n=84)$ n'ont pas d'avis sur le mode d'exercice souhaité.

\section{Analyse de la relation entre les caractéristiques individuelles des répondants et le fait d'avoir rempli le dernier formulaire}

Parmi les 223 répondants, 186 étudiants ont répondu avoir renvoyé le dernier formulaire d'évaluation de stage et 37 étudiants ont répondu ne pas l'avoir envoyé. Les résultats de l'analyse de la relation entre les caractéristiques individuelles des répondants et le fait d'avoir rempli le dernier formulaire sont présentés dans le tableau I.

Les étudiants n'ayant pas rempli le dernier formulaire d'évaluation exercent plus fréquemment une activité rémunérée $(p<0,008)$ et souhaitent avoir plus fréquemment un exercice de type salarié que les étudiants ayant rempli le dernier formulaire avec une différence statistiquement significative.

Les résultats de l'analyse multivariée, présentés dans le tableau II, mettent en évidence que les deux facteurs «absence d'activité rémunérée » et «mode d'exercice envisagé de type salarié » sont des déterminants significatifs avec un odds ratio respectivement de 0,21 et 0,41. Cela signifie que la probabilité de remplir le dernier formulaire d'évaluation par les étudiants ayant une activité rémunérée est cinq fois moindre que pour les autres étudiants et que la probabilité de remplir le dernier formulaire en ligne par les étudiants qui désirent exercer une activité salariale est 2,4 fois moindre que pour les autres étudiants. 
Tableau I. Nombres de réponses positives aux items du « questionnaire portant sur les caractéristiques individuelles » analysés de manière comparative entre les répondants ayant rempli le dernier formulaire et les répondants n'ayant pas rempli le dernier formulaire.

\begin{tabular}{llccccc}
\hline Variables qualitatives & & $\begin{array}{c}\text { Répondants ayant } \\
\text { rempli le dernier } \\
\text { formulaire } \\
\end{array}$ & $\begin{array}{c}n=186 \\
\text { Répondants n'ayant } \\
\text { pas rempli le dernier } \\
\text { formulaire } \\
n=37\end{array}$ & $\boldsymbol{p}$ \\
\hline \multirow{2}{*}{ Sexe } & Homme & $\boldsymbol{n}$ & $\mathbf{\%}$ & $\boldsymbol{n}$ & $\%$ & \\
& Femme & 60 & 32,3 & 15 & 40,5 & $\mathrm{~ns}$ \\
Étudiant redoublant & & 126 & 67,7 & 22 & 59,5 & $\mathrm{~ns}$ \\
Activité rémunérée & & 5 & 2,7 & 1 & 2,7 & $\mathrm{~ns}$ \\
Accès internet au domicile & & 14 & 7,5 & 8 & 21,6 & 0,015 \\
Connexion wifi & & 168 & 90,3 & 30 & 81,1 & $\mathrm{~ns}$ \\
Participation aux confé- & & 126 & 67,7 & 23 & 62,2 & $\mathrm{~ns}$ \\
rences d'internat & & 106 & 57 & 18 & 48,6 & $\mathrm{~ns}$ \\
Type de logement & Chez les parents & 30 & 16,1 & 1 & 2,7 & $\mathrm{~ns}$ \\
& Seul & 97 & 52,2 & 21 & 56,8 & $\mathrm{~ns}$ \\
Type de spécialité & Colocation/cité universitaire & 59 & 31,7 & 15 & 40,5 & $\mathrm{~ns}$ \\
envisagé & Médecine générale & 49 & 26,3 & 5 & 13,5 & $\mathrm{~ns}$ \\
& Spécialité médicale & 78 & 41,9 & 13 & 35,1 & $\mathrm{~ns}$ \\
Mode d'exercice envisagé & Spécialité chirurgicale & 21 & 11,3 & 7 & 18,9 & $\mathrm{~ns}$ \\
& Libéral & 88 & 47,3 & 14 & 37,8 & $\mathrm{~ns}$ \\
& Salarié & 23 & 12,4 & 14 & 37,8 & 0,0006 \\
& Sans avis & 75 & 40,3 & 9 & 24,3 & $\mathrm{~ns}$ \\
\hline
\end{tabular}

Tableau II. Résultats de la régression logistique avec les variables « activité rémunérée » et « mode d'exercice envisagé de type salarié ".

\begin{tabular}{lccc}
\hline Variable & $\boldsymbol{p}$ & Odds ratio & IC OR (95 \%) \\
\hline $\begin{array}{l}\text { Activité rémunérée } \\
\begin{array}{l}\text { Mode d'exercice envisagé de type } \\
\text { salarié }\end{array}\end{array}$ & 0,001 & 0,216 & {$[0,077-0,607]$} \\
\hline IC OR : & 0,0006 & 0,416 & {$[0,244-0,708]$} \\
\hline
\end{tabular}

IC OR : intervalle de confiance sur l'odds ratio.

\section{Analyse de la relation entre les caractéristiques individuelles des répondants et le fait d'avoir rempli tous les formulaires en ligne en 2008-2009}

Parmi les 223 répondants, on compte 141 étudiants déclarant avoir rempli tous les formulaires en ligne au cours de l'année précédente, 27 étudiants ayant répondu avoir rempli plus de $50 \%$ des formulaires et 16 étudiants ayant répondu avoir rempli moins de $50 \%$ des formulaires au cours de l'année précédente.
Le nombre de non répondants à cette question s'élève à $n=39$.

Les résultats de l'analyse de la relation entre les caractéristiques individuelles des répondants et le fait d'avoir rempli tous les formulaires en ligne sont présentés dans le tableau III.

Les étudiants n'ayant pas rempli tous les formulaires d'évaluation en 2008-2009 sont en moyenne plus âgés (âge $=23,4$ année $\pm 1,36$ ) que ceux ayant 
Tableau III. Nombres de réponses positives aux items du " questionnaire portant sur les caractéristiques individuelles » analysées de manière comparative entre les répondants ayant rempli tous les formulaires et les autres répondants.

\begin{tabular}{|c|c|c|c|c|c|c|}
\hline \multirow[t]{2}{*}{ Variables qualitatives } & & \multicolumn{2}{|c|}{$\begin{array}{l}\text { Répondants } \\
\text { ayant rempli } \\
\text { tous les } \\
\text { formulaires } \\
n=141\end{array}$} & \multicolumn{2}{|c|}{$\begin{array}{c}\text { Répondants } \\
\text { n'ayant pas rempli } \\
\text { tous les } \\
\text { formulaires } \\
n=43\end{array}$} & \multirow[t]{2}{*}{$p$} \\
\hline & & $n$ & $\%$ & $n$ & $\%$ & \\
\hline \multirow[t]{2}{*}{ Sexe } & Homme & 49 & 34,8 & 14 & 32,6 & ns \\
\hline & Femme & 92 & 65,2 & 29 & 67,4 & ns \\
\hline Étudiant redoublant & & 3 & 2,1 & 2 & 4,7 & ns \\
\hline Activité rémunérée & & 9 & 6,4 & 5 & 11,6 & ns \\
\hline Accès internet au domicile & & 127 & 90,1 & 39 & 90,7 & ns \\
\hline Connexion wifi & & 98 & 69,5 & 27 & 62,8 & ns \\
\hline $\begin{array}{l}\text { Participation aux } \\
\text { conférences d'internat }\end{array}$ & & 86 & 61,0 & 19 & 44,2 & 0,04 \\
\hline \multirow[t]{3}{*}{ Type de logement } & Chez les parents & 22 & 15,6 & 7 & 16,3 & ns \\
\hline & Seul & 76 & 53,9 & 21 & 48,8 & $\mathrm{~ns}$ \\
\hline & Colocation/cité universitaire & 43 & 30,5 & 15 & 34,9 & ns \\
\hline \multirow[t]{3}{*}{ Type de spécialité envisagé } & Médecine générale & 34 & 24,1 & 14 & 32,6 & ns \\
\hline & Spécialité médicale & 76 & 53,9 & 22 & 51,2 & ns \\
\hline & Spécialité chirurgicale & 17 & 12,1 & 3 & 7,0 & ns \\
\hline \multirow[t]{3}{*}{ Mode d'exercice envisagé } & Libéral & 67 & 47,5 & 19 & 44,2 & ns \\
\hline & Salarié & 17 & 12,1 & 7 & 16,3 & $\mathrm{~ns}$ \\
\hline & Sans avis & 57 & 40,4 & 17 & 39,5 & ns \\
\hline
\end{tabular}

Tableau IV. Résultats de la régression logistique avec les variables « âge » et « participation aux conférences d'internat».

\begin{tabular}{lccc}
\hline Variable & $\boldsymbol{p}$ & Odds ratio & IC OR (95 \%) \\
\hline Age & 0,141 & 0,915 & {$[0,778-1,076]$} \\
Participation aux conférences d'internat & 0,019 & 2,136 & {$[1,041-4,388]$} \\
\hline
\end{tabular}

IC OR : intervalle de confiance sur l'odds ratio.

rempli tous les formulaires (âge $=23,16$ année $\pm 2,12$ ) avec une différence statistiquement significative $(p<0,017)$.

Les étudiants n'ayant pas rempli tous les formulaires d'évaluation en 2008-2009 participent moins fréquemment aux conférences d'internat $(p<0,04)$.

Compte tenu des précédents résultats, l'existence éventuelle d'une corrélation entre l'âge des étudiants et la participation aux conférences d'internat a été recherchée. Il s'avère que les étudiants participant aux conférences d'internat sont plus âgés, sans différence statistiquement significative. Les deux facteurs « âge » et « participation aux conférences d'internat » ne sont donc pas liés.

Quoi qu'il en soit, la différence d'âge observée entre les deux groupes de 0,24 années - soit 2,8 mois - ne correspond pas à un résultat cliniquement pertinent.

Les résultats de l'analyse multivariée, présentée dans le tableau IV, mettent en évidence que le seul 
déterminant significatif est «la participation aux conférences d'internat» avec un odds ratio de 2,13. Cela signifie que la probabilité de remplir tous les formulaires par les étudiants assistant aux conférences est doublée par rapport aux étudiants n'y assistant pas.

\section{Questionnaire de motivation}

\section{Motivation des répondants ayant rempli le dernier formulaire}

Parmi les étudiants ayant rempli le dernier formulaire ( $n=186)$, on constate que la majorité déclare l'avoir fait pour donner leur avis $(n=156 ; 83,8 \%)$ et pour que le stage soit validé par la faculté de médecine $(n=150 ; 80,6 \%)$. En revanche l'incitation par les délégués étudiants n'a pas eu d'influence $(n=16$; $8,6 \%$ ).

Les autres raisons citées par les étudiants en réponse libre sont le désir de signaler « un bon stage » $(n=4 ; 2,1 \%)$, le désir de signaler « un mauvais stage » $(n=3 ; 1,6 \%)$ et le fait d'avoir reçu un mail de rappel $(n=3 ; 1,6 \%)$.

\section{Raisons évoquées par les répondants n'ayant pas rempli le dernier formulaire}

Parmi les étudiants n'ayant pas rempli le dernier formulaire $(n=36)$ la principale raison retrouvée est «l'oubli » $(n=18 ; 50 \%)$.

Les autres raisons citées par les étudiants dans les réponses libres sont « un temps d'ouverture des votes trop court » $(n=2 ; 5,5 \%)$ et « la non-réception du mail d'invitation » $(n=7 ; 19,4 \%)$.

\section{Raisons pour lesquelles l'évaluation est jugée inutile par les répondants}

Parmi les 69 étudiants (soit 1/3 des répondants) qui trouvent que l'évaluation est inutile, les trois principales raisons invoquées sont que « les résultats ne sont pas accessibles aux étudiants » $(n=49 ; 71 \%)$, que «les stages sont toujours aussi mal organisés» $(n=61 ; 88,4 \%)$ et enfin que « la faculté n'a pas les moyens d'obliger les services hospitaliers à améliorer les stages » $(n=57 ; 82,6 \%)$.
Les raisons citées dans les réponses libres rejoignent ces items et insistent notamment sur l'absence de répercussion de l'évaluation sur la qualité des stages.

\section{Analyse de la relation entre les réponses au questionnaire de motivation et le fait d'avoir rempli tous les formulaires d'évaluation des stages en 2008-2009}

Aucune corrélation n'a pu être établie.

\section{Discussion}

Si la littérature nationale compte plusieurs études portant sur l'évaluation des enseignements cliniques ou théoriques ${ }^{[5,6]}$, aucune étude ne s'était intéressée jusqu'à présent à la motivation des étudiants à participer à l'évaluation des enseignements. Or, au regard du faible taux de retour des formulaires d'évaluation des stages par les étudiants du deuxième cycle à la faculté de médecine de Strasbourg, il semblait important de s'intéresser à cette problématique.

Définition des caractéristiques individuelles des étudiants ayant rempli le dernier formulaire d'évaluation

Compte tenu des résultats de l'enquête, les caractéristiques individuelles des non-répondants diffèrent selon qu'il s'agit des non-répondants au dernier formulaire ou des non-répondants à tous les formulaires. Ainsi, on s'aperçoit que la probabilité de remplir le dernier formulaire d'évaluation est cinq fois moindre pour un étudiant qui a une activité rémunérée. L'hypothèse serait alors que l'exercice d'une activité rémunérée réduirait la disponibilité des étudiants. À titre comparatif, une étude réalisée par l'observatoire national de la vie étudiante (OVE) en 2000 sur 26000 étudiants inscrits à l'université, montre que lorsque l'activité rémunérée non intégrée aux études est pratiquée au moins à mi-temps, les probabilités de réussite totale diminuent de $29 \%{ }^{[7,8]}$. Certes, cette enquête ne porte pas sur la réussite aux examens, mais 
les résultats de l'étude rejoignent l'hypothèse selon laquelle l'activité rémunérée constituerait un frein à la disponibilité de l'étudiant pour ses études.

La probabilité de remplir le dernier formulaire d'évaluation est également 2,4 fois moindre pour les étudiants ayant un projet salarié. Ce résultat est surprenant car on aurait davantage pu s'attendre au résultat inverse à savoir que les étudiants envisageant une activité libérale soient amenés à s'éloigner de la faculté et donc des préoccupations liées à l'amélioration des pratiques de formation.

De plus, le fait d' assister aux conférences d'internat double la probabilité de répondre à l'ensemble des formulaires. Un tel résultat pourrait être expliqué par le fait que les répondants à tous les formulaires soient davantage impliqués dans l'amélioration pédagogique des stages hospitaliers.

Compte tenu de l'absence de lien retrouvé par les analyses statistiques entre le taux de réponse et le contexte de vie de l'étudiant, l'hypothèse formulée initialement selon laquelle d'éventuelles contraintes matérielles (type de logement, accessibilité à internet) pouvait avoir un impact sur le taux de réponse n'a pas été validée. Par conséquent aucune piste d'action portant sur le contexte de vie de l'étudiant n'a pu être identifiée. Le mode de recueil des formulaires par internet n'a notamment pas été mis en cause.

\section{Profil motivationnel des répondants}

Le profil motivationnel des étudiants ayant rempli le dernier formulaire en ligne est fortement marqué par le sentiment d'agir sous la contrainte (menace de non-validation de stage par la faculté en cas de nonréponse), ce qui témoigne d'une motivation extrinsèque de type externe, associée à un niveau d'autodétermination faible.

De plus les étudiants qui ne répondent pas à l'évaluation ont une perception de la valeur de la tâche et une perception de contrôle très faibles. Or, au regard des approches théoriques exposées précédemment, il existe une corrélation positive entre la perception de la valeur de la tâche, la perception de contrôle et la motivation intrinsèque ${ }^{[9-11]}$. En d'autres termes, une perception élevée de la valeur de la tâche et de contrôle est préférentiellement associée à des comportements intrinsèquement motivés ${ }^{[12,13]}$.

Par conséquent, afin d'améliorer l'implication des étudiants dans l'évaluation des stages hospitaliers, des actions visant à améliorer la perception de la valeur de la tâche et la perception de contrôle pourraient être envisagées. En s'inspirant des propositions décrites dans des études antérieures ${ }^{[12,14]}$, les actions envisageables dans cette étude se situent à différents niveaux :

- les buts de l'évaluation pourraient être annoncés et expliqués au début du formulaire d'évaluation en ligne ;

- la campagne d'information des étudiants débutée avant cette enquête à l'aide d'affiches sur les enjeux de l'évaluation et l'importance de leur point de vue devra être poursuivie ;

- les résultats et les propositions d'amélioration formulées grâce à cette enquête devraient être présentés aux étudiants ;

- la diffusion des résultats des évaluations auprès des étudiants, débutée pour la première fois au mois de mars 2010, devrait être poursuivie permettant ainsi un «feedback» ou rétroaction positive pour aider les étudiants dans leur cheminement d'apprentissage ;

- les propositions d'améliorations ou de changements pourraient être discutées au sein de la CEETC en présence des référents pédagogiques et des représentants des étudiants, avant d'être diffusées aux étudiants. Si pour des raisons quelconques, les changements souhaités ne peuvent se matérialiser dans un avenir rapproché, il sera tout aussi important d'en informer les étudiants et d'en expliquer les raisons, pour maintenir leur motivation ${ }^{[6]}$.

Au-delà des actions à entreprendre auprès des étudiants, il nous semble également important d'identifier les raisons qui motivent les enseignants à modifier leur pratique en tenant compte des résultats de l'évaluation des stages. En effet, la concrétisation des propositions d'amélioration des stages se réalisera d'autant plus facilement que le niveau d'auto-détermination des enseignants à modifier leur pratique sera élevé. La perception des professeurs cliniciens de l'évaluation des enseignements théoriques a déjà fait l'objet d'une étude par Normand et Bernard ${ }^{[15]}$. Dans 
cette enquête, les cliniciens perçoivent l'évaluation de l'enseignement comme une opération utile, susceptible de les aider à s'améliorer. Les cliniciens émettent également le souhait d'avoir accès à des ressources pour améliorer leur enseignement. Au-delà de la perception de l'évaluation de l'enseignement, une évaluation de la motivation des enseignants à l'application des propositions d'amélioration des stages émanant de la CEETC permettrait d'identifier le profil motivationnel des enseignants, afin d'améliorer la concordance entre les attentes des apprenants et celles des enseignants.

Limites de l'étude

\section{Choix méthodologique}

Le choix d'une démarche quantitative avec une collecte de données par questionnaire auto-administré a été motivé par des questions de faisabilité. En effet, compte tenu du temps qui nous était imparti et du nombre limité de chercheurs impliqués dans ce travail, cette méthode de recueil d'informations nous semblait la plus pertinente.

De même, le choix du format des questions de type OUI/NON a été préféré à l'échelle de Likert, habituellement utilisée dans les questionnaires de ce type, essentiellement pour des raisons de logistique. En effet, la deuxième partie du questionnaire intitulé «questionnaire de motivation» comporte à la fois des questions dont les réponses peuvent être nuancées et des questions dont seule une réponse OUI/NON peut être donnée. De ce fait, il s'avérait plus simple de privilégier un seul format de question afin de faciliter le codage et l'analyse statistique.

Cependant, cette étude préliminaire pourrait être enrichie par une démarche qualitative, impliquant un recueil de données sur la base, par exemple, d'entretiens individuels ou de groupes de discussion focalisée (focus group). L' analyse qualitative permettrait notamment de définir de nouvelles pistes de recherche auxquelles nous n'aurions pas pensé ou, encore, d'explorer plus en profondeur certains éléments du profil motivationnel tels que les attentes des étudiants vis-à-vis de cette démarche d'évaluation des stages.

\section{Élaboration du questionnaire}

Si la fiabilité du questionnaire a été évaluée par le calcul de sa cohérence interne, la validité de construit du questionnaire ne peut être considérée comme établie, ce qui constitue la principale limite notre étude. Par conséquent, en complément de la démarche qualitative abordée précédemment, nous pourrions envisager une nouvelle étude quantitative avec un nouveau questionnaire s'appuyant sur les questionnaires motivationnel préexistants ${ }^{[16]}$. Au terme de ce travail, nous pourrions ainsi disposer d'un instrument francophone solide adapté à l'évaluation des facteurs motivationnels des étudiants en médecine à l'égard des questionnaires d'évaluation des stages.

\section{Limites inhérentes aux questionnaires sur la motivation et aux questionnaires en général}

Comme tous les questionnaires explorant le registre de la motivation, le questionnaire utilisé dans cette étude a une puissance de mesure limitée, car les items relatifs à l'image de soi exigent une réflexion métacognitive que certains sujets ne seront probablement pas disposés ou capables de produire ${ }^{[16]}$.

L'utilisation des questionnaires en général soustend également des limites ${ }^{[17]}$. En effet, les répondants peuvent être amenés à déformer volontairement leurs propos, et ce même si le questionnaire est anonyme, pour des raisons diverses : vouloir donner une image positive de soi ou de sa profession ; ne pas souhaiter révéler des comportements moralement répréhensibles ou éthiquement condamnables ; essayer stratégiquement d'influencer les conséquences opportunément perçues de l'étude réalisée.

\section{Taux de réponse}

La population cible à laquelle le questionnaire s'adressait était la population des étudiants inscrits en DCEM3 à la faculté de médecine de Strasbourg en 2009-2010, soit 279 étudiants. Il a été fixé comme critère d'exclusion les étudiants Erasmus. L'ensemble des étudiants, présents aux travaux dirigés du module de médecine légale durant l'année universitaire 20092010 auxquels le questionnaire a été soumis, a constitué l'effectif de la population d'étude à savoir 
223 sujets. Eu égard à l'absence de 56 étudiants au sein de l'effectif comparativement au nombre total d'étudiants inscrits en DCEM3, on peut se questionner sur la représentativité de l'échantillon d'étude par rapport à la totalité des étudiants de la promotion. Par conséquent, les conclusions de cette étude porteront uniquement sur l'échantillon d'étude et devront être généralisées de manière prudente à l'ensemble de la promotion DCEM3.

\section{Conclusion}

Connaître les motifs de l'implication active des étudiants dans l'évaluation en ligne des stages hospitaliers est indispensable dans une démarche d'évaluation de la qualité de ces stages.

L'identification du profil motivationnel des étudiants ayant rempli le dernier formulaire d'évaluation en ligne a permis de mettre en évidence le caractère faiblement autodéterminé des motifs d'engagement des étudiants dans l'évaluation.

L'analyse de la dynamique motivationnelle a permis de souligner la faible perception de la valeur de la tâche et la faible perception de contrôle ressentie par les étudiants. La connaissance de ces données a permis de formuler des propositions concrètes d'actions afin d'augmenter le niveau d'autodétermination des étudiants dans le processus d'évaluation des stages. L'identification des raisons motivant les enseignants à modifier leur pratique en tenant compte des résultats de l'évaluation de stages constitue la prochaine étape s'inscrivant dans le cadre de la démarche qualité entreprise en sein de la faculté de médecine de Strasbourg.

\section{Contributions}

Audrey Farrugia, Thierry Pottecher et Bertrand Ludes ont conjointement mené des discussions sur le contenu et la structuration de l'article. Thierry Pelaccia a élaboré le cadre théorique. Thierry Pottecher a effectué les analyses statistiques. Le manuscrit a été révisé et validé par l'ensemble des auteurs.

\section{Annexe 1 : Questionnaire utilisé}

I. Questionnaire portant sur les caractéristiques individuelles

\author{
Votre âge : \\ Votre sexe : $\square \mathrm{F} \quad \square \mathrm{M}$ \\ Êtes-vous redoublant de D3 : \\ $\square$ OUI $\square$ NON
}

Avez-vous une activité rémunérée (en dehors des stages) pour financer vos études :

\section{$\square$ OUI $\square$ NON}

Avez-vous un accès quotidien à internet à votre domicile :

\section{$\square$ OUI $\square$ NON}

Avez-vous un ordinateur portable avec une connexion wifi :

$\square$ OUI $\square$ NON

Vous habitez en semaine :

$\square$ chez vos parents

$\square$ dans un studio/appartement seul(e)

$\square$ en coloc

$\square$ en cité U

Participez-vous à une conf. de préparation à l'ECN $\square$ OUI $\square$ NON

Si vous êtes bien classé(e) à l'ECN, vous vous destinez à :

$\square$ La médecine générale :

en libéral $\square$, comme salarié $\square$ ne sait pas $\square$

$\square$ Une autre spécialité médicale :

en libéral $\square$, comme salarié $\square$ ne sait pas $\square$

$\square$ Une spécialité chirurgicale :

en libéral $\square$, comme salarié $\square$ ne sait pas $\square$

II. Questionnaire de motivation (la phrase en italique après chaque question est une reformulation de la question pour en faciliter sa compréhension)

Avez-vous rempli le formulaire d'évaluation en ligne après votre dernier stage hospitalier ? $\square$ OUI $\square$ NON

\section{Si votre réponse est OUI}

Vous l'avez fait car il vous semble important de donner votre avis "pour améliorer les choses dans les futurs stages »

\section{$\square$ OUI $\quad \square$ NON}

Vous l'avez fait car le système est simple et convivial «facile d'utilisation et rapide »
$\square$ OUI
$\square \mathrm{NON}$ 
Vous l'avez fait car ce formulaire peut être rempli de votre domicile «gain de temps/choix $d u$ moment »

\section{$\square$ OUI $\quad \square$ NON}

Vous l'avez fait, incité(e) par vos délégués étudiants «encouragé/motivé »

\section{$\square$ OUI $\quad \square$ NON}

Vous l'avez fait pour que votre stage soit validé par la Fac «obligation administrative »

$\square$ OUI $\quad \square$ NON

Autres raisons, précisez :

Avez-vous rempli d'autres formulaires d'évaluation en ligne pendant l'année 2008-09 ?

$$
\square \text { tous } \quad \square \geq 50 \% \quad \square<50 \%
$$

\section{Si votre réponse est NON}

Vous ne pouvez pas consulter les mails envoyés par la Fac de Médecine "pas d'accès internet » $\square$ OUI $\quad \square$ NON

Il n'est pas spécifié sur le mail d'invitation qu'il vient de la faculté (aspect de Spam) « destinataire non précisé»

\section{$\square$ OUI $\quad \square$ NON}

Vous êtes contre l'évaluation en général et ne remplissez jamais aucun formulaire (sauf bien sûr celui-ci !)

\section{$\square$ OUI $\quad \square$ NON}

Vous n'avez pas eu accès à Internet pendant la période d'ouverture des votes

\section{$\square$ OUI $\quad \square$ NON}

Vous avez oublié de le remplir alors que vous le faites habituellement "pas le temps, trop occupé»

\section{$\square$ OUI $\quad \square$ NON}

Vous trouvez ce questionnaire trop compliqué «ambiguë, incompréhensible »

\section{$\square$ OUI $\quad \square$ NON}

Vous trouvez que la procédure d'évaluation en ligne des stages est trop chronophage
$\square$ OUI
$\square$ NON

Autres raisons, précisez :

\section{Dans tous les cas :}

Vous pensez que l'évaluation des stages est inutile $\square$ OUI $\quad \square$ NON

Si vous avez répondu oui à cette dernière question, c'est :

- parce que les résultats ne sont pas accessibles aux étudiants «non diffusés au étudiants »

\section{$\square$ OUI $\quad \square$ NON}

- parce que depuis que l'évaluation a été instaurée, les stages sont toujours aussi mal organisés $\square$ OUI $\quad \square$ NON

- parce que la faculté n'a pas les moyens d'obliger les services hospitaliers à améliorer les stages (encadrement et organisation)

$\square$ OUI $\quad \square$ NON

- parce que vous trouvez « impoli » d'évaluer le travail de vos aînés «manque de respect »

$\square$ OUI $\quad \square$ NON

- pour d'autres raisons, précisez :

\section{Références}

1. Vallerand RJ, Thill EE. Introduction au concept de motivation. In : Vallerand RJ, Thill EE (eds). Introduction à la psychologie de la motivation. Laval (Québec) : Éditions Études Vivantes, 1993:3-39.

2. Deci EL, Ryan RM. Intrinsic motivation and selfdetermination in human behavior. New York: Edition Plenum Press, 1985.

3. Pelaccia T, Triby E, Delplancq H, Bartier J-C, Ammirati $\mathrm{C}$, Leman $\mathrm{C}$ et al. Quels sont les déterminants de la motivation des étudiants et des professionnels de santé en formation aux gestes et soins d'urgence ? Étude comparative du profil motivationnel des étudiants en médecine, des chirurgiens-dentistes libéraux et des infirmiers hospitaliers. Pédagogie Médicale 2009;10:83-94.

4. Viau R. La motivation en contexte scolaire (Édition européenne). Bruxelles : De Boeck ( $2^{\mathrm{e}}$ édition révisée), 2009.

5. Ploteau S, N Winer N, Barrier J, Rogez J-M, Philippe H-J. Évaluation des enseignements et des enseignants par les externes lors d'un stage clinique dans un service de gynécologie-obstétrique. J Gynecol Obstet Biol Reprod 2007;36:807-16. 
6. Barrier J-H, Balde N, Brazeau-Lamontagne L, Normand S, Essoussi AS, Fiche M et al. L'évaluation de l'enseignement : pour quelles décisions ? Pédagogie Médicale 2006;4:238-47.

7. Thiphaine B. Les étudiants et l'activité rémunérée. Observatoire National de la Vie étudiante Infos, 2002 ;1. [On-line] Disponible sur http://www.ove national.education.fr/medias/files/ove-infos/ oi1_oi1.pdf

8. Gruel L. Les conditions de réussite dans l'enseignement supérieur. Observatoire National de la Vie étudiante Infos, 2002;2. [On-line] Disponible sur http:/ /www.ove-national.education.fr/medias/files/ ove-infos/oi2_oi2.pdf

9. Pelaccia T, Delplancq H, Triby E, Bartier J-C, Leman C, Dupeyron J-P. Impact of training periods in the emergency department on the motivation of health care students to learn. Med Educ 2009;43:462-9.

10. Husman J, Derryberry WP, Crowson HM, Lomax R. Instrumentality, task value, and intrinsic motivation: Making sense of their independent interdependence. Contemp Educ Psychol 2004;29:63-76.

11. Pintrich P-R, Smith D, Garcia T. et McKeachie W-J. A manual for the use of the motivated strategies for learning questionnaire (MSLQ). Ann Arbor: University of Michigan (National Center for Research to Improve Postsecondary Teaching and Learning) 1991.
12. Pelaccia T, Delplancq H, Triby E, Leman C, Bartier J-C, Dupeyron J-P. La motivation en formation : une dimension réhabilitée dans un environnement d'apprentissage en mutation. Pédagogie Médicale 2008;9:103-21.

13. Weiner B. Motivation from an attribution perspective and the social psychology of perceived competence. In: Elliot AJ, Dweck CS (eds). Handbook of competence and motivation. New-York: Guilford Press, 2005:73-84.

14. Pelaccia T, Delplancq H, Triby E, Leman C, Bartier $\mathrm{J}-\mathrm{C}$, Brunstein $\mathrm{V}$ et al. 10 recommendations to integrate students' motivation in teaching and assessment strategies. In: Wergers CE(eds). Nursing students and their concerns. New-York: Novascience, 2010.

15. Normand S, Bernard H. Les professeurs cliniciens se prononcent sur l'évaluation de l'enseignement. Pédagogie Médicale 2002;4:202-9.

16. Pintrich, PR and others. A Manual for the use of the motivated strategies for learning questionnaire (MSLQ), 1991.

17. Aubert J. Motivations et ressources humaines. Education permanente 1993;136:163-171.

18. Angers M. Initiation pratique à la méthodologie des sciences humaines. Montréal : Éditions CEC, 1996.

Correspondance et offprints : Audrey Farrugia, Institut de Médecine légale, 11 rue Humann, 67085 Strasbourg Cedex, France.

Mailto: audrey.farrugia@unistra.fr 